\title{
Efecto de la anestesia epidural sobre el flujo de las arterias mamarias en cirugía cardíaca
}

\author{
Sánchez DN. ${ }^{1}$, Felici S. ${ }^{1}$, Renna N. ${ }^{2}$ \\ 1 Hospital Central de Mendoza, Mendoza, Argentina. \\ 2 Área de Fisiología Patológica. Facultad de Ciencias Médicas. U.N. Cuyo, Mendoza, Argentina.
}

Introducción: La anestesia epidural (AE), se usa hace más de 20 años para este tipo de intervenciones, y genera numerosos beneficios, tales como excelente analgesia post-operatoria, disminución del tiempo de asistencia respiratoria mecánica, disminución de la estadía hospitalaria, mejoría de la función pulmonar, disminución de la respuesta al stress, etc.

El objetivo de este trabajo es investigar el impacto de esta técnica en pacientes sometidos a cirugía de revascularización miocárdica, sobre todo en lo que respecta al flujo de ambas arterias mamarias, desde un punto de vista anatómico y fisiológico. Es importante destacar que mantener un flujo adecuado en las arterias mamarias es fundamental para la irrigación del miocardio revascularizado.

Material y Método: El siguiente trabajo de investigación, se llevó a cabo en el Servicio de Cirugía Cardíaca del Hospital Central de Mendoza, en forma prospectiva y aleatorizada. Los participantes fueron sometidos a cirugía de revascularización miocárdica, con doble injerto arterial (con la utilización de Arteria mamaria izquierda AMI, y derecha AMD), y sin la utilización de la bomba de circulación extracorpórea. La totalidad de los participantes fueron informados del estudio y firmaron el consentimiento informado aprobado por el Comité de Ética del Hospital Central de Mendoza.

Los participantes fueron asignados de manera aleatoria en dos grupos de treinta participantes. El grupo 1 (uno), correspondiente a los pacientes que solo recibieron anestesia general para la cirugía y el grupo 2 (dos), los cuales recibieron anestesia general más anestesia epidural para la cirugía.

Análisis estadístico: ANOVA de una vía.

Resultados:

1)Se realizó el análisis de los flujos de sangre de las arterias mamarias de ambos grupos. El grupo número 1 (sin anestesia epidural) mostró los siguientes resultados 9,31 $\pm 5,5 / 12,4 \pm 6,0$ (AMI/AMD), versus los valores del grupo del grupo 2 (con epidural) 17,43 $\pm 6,8 / 18,8 \pm 5,9$ (AMI/AMD). El análisis estadístico posterior mostró una $p<0,001$.

2)En el análisis histológico de cortes de tejido de AMI y AMD con tinción de hematoxilina-eosina y tricrómico de masson en ambos grupos, no mostraron diferencia significativa de la relación lumen/media.

Conclusiones:

1)La técnica de anestesia general más epidural, fue altamente efectiva para aumentar el flujo de ambas arterias mamarias, siendo esto muy importante para optimizar la revascularización del miocardio.

2)Se esperan cambios más agudos en las arterias mamarias, por lo cual estudiaremos los cortes obtenidos mediante inmunohistoquimica buscando receptores de inflamación.

https://doi.org/10.25237/congresoclasa2019.70 